ESAIM: COCV 21 (2015) 399-413

DOI: $10.1051 / \mathrm{cocv} / 2014030$
ESAIM: Control, Optimisation and Calculus of Variations

www.esaim-cocv.org

\title{
THE NORM OPTIMAL CONTROL PROBLEM FOR STOCHASTIC LINEAR CONTROL SYSTEMS*
}

\author{
YANQING WANG ${ }^{1}$ AND CAN ZHANG ${ }^{2, * *}$
}

\begin{abstract}
In this paper we are concerned with two norm optimal control problems for different stochastic linear control systems. One is for approximately controllable systems with the natural filtration, while another is for exactly controllable systems with a general filtration. For each aforementioned norm optimal control problem, we construct the unique norm optimal control, through building up some suitable quadratic functional and making use of a variational characterization on its minimizer.
\end{abstract}

Mathematics Subject Classification. 93E20, 93C05.

Received January 19, 2013. Revised April 21, 2014.

Published online January 15, 2015.

\section{INTRODUCTION}

Let $(\Omega, \mathcal{F}, \mathbb{F}, P)$ be a complete filtered probability space (satisfying the usual conditions), on which a standard 1-dimensional Brownian motion $\{W(t) ; t \geq 0\}$ is defined. Let $T>0$. Consider the following stochastic linear control system (with suitable coefficient matrices $F, G, F_{1}$ and $G_{1}$ ):

$$
\left\{\begin{aligned}
\mathrm{d} y(t) & =(F y(t)+G u(t)) \mathrm{d} t+\left(F_{1} y(t)+G_{1} u(t)\right) \mathrm{d} W(t), \quad t \in[0, T] \\
y(0) & =y_{0} .
\end{aligned}\right.
$$

In (1.1), $y_{0} \in \mathbf{R}^{n}(n \in \mathbf{N})$; while $u(\cdot)$ and $y(\cdot)$ are respectively the control and state variables. Suppose this system is controllable (which will be defined precisely later). Then, for any target set, there exists a control process $u(\cdot)$ steering the state process $y(\cdot)$ from any given $y_{0}$ at the initial time $t=0$ to the target set at some specified time. We call such a control an admissible control. The purpose of this paper is to find a control $\hat{u}(\cdot)$ with the minimal norm among all admissible controls. Such a control is called a norm optimal control.

The controllability for the finite-dimensional deterministic linear control systems is completely characterized by the well-known Kalman algebraic criterion. However, for stochastic control systems, the situation is much

Keywords and phrases. Norm optimal control, stochastic linear control systems, controllability, filtration.

* Part of this work was finished when this author was a Ph.D. student at the "Key Laboratory of Systems and Control, Academy of Mathematics and Systems Science, Chinese Academy of Sciences". This author is supported by the National Basic Research Program of China (973 Program) under grant 2011CB808002, by the NSF of China under Grants 11231007 and 11101452 and by Fundamental Research Funds for the Central Universities under grants SWU113038 and XDJK2014C076.

1 School of Mathematics and Statistics, Southwest University, Chongqing 400715, P.R. China. yqwang@amss.ac.cn

2 School of Mathematics and Statistics, Wuhan University, Wuhan 430072, P.R. China. canzhang@whu.edu.cn

** This author is supported by the NSF of China under Grants 11161130003 and 11171264. 
less satisfactory and there have been very limited publications $(c f .[2,16])$ about the controllability before 1990. With the aid of backward stochastic differential equations (BSDEs, for short), some kinds of controllability have been derived. In [11], the author defined the stochastic exact controllability for the system (1.1) with natural filtration, and characterized it by an algebraic condition of Kalman-type. For the systems of jump diffusions with general filtration, the exact controllability in the transposition sense was defined in [14] and the corresponding Kalman-type condition was also established. For the stochastic approximate controllability of the finite-dimensional systems and the related generalized Kalman-type condition, we refer to $[1,5]$ (without jump diffusions) and [6] (with jump diffusions).

In the deterministic case, the existence of norm optimal controls is guaranteed by the controllability of the corresponding system. In this case, the norm optimal control can be characterized by either the Pontryagin maximum principle (cf. e.g. [4]) or a minimizer of some quadratic functional (cf. e.g. [13, 17]), for which the desired coercivity is easily verified because the deterministic differential equation is invertible with respect to the time variable. In this study, we adopt the minimization method (cf. e.g. $[13,17]$ ) to obtain sufficient and necessary conditions for the solvability of the norm optimal control problem for linear stochastic control systems. We will face to a BSDE, instead of the time-invertible differential equation. We successfully pass this barrier to prove the coercivity of a similar quadratic functional by using the unique continuation property for solutions to BSDEs.

The norm optimal control problem considered in this work can be viewed as a stochastic control problem with some terminal state constraints. The later has been studied in $[7,8,15]$. In [7], a forward-backward stochastic differential equation controlled system is re-formulated as a purely backward system by treating the terminal condition of the forward state as a "control". By this way, a stochastic Pontryagin maximum principle is derived by means of Ekeland's variational principle. In [8], a stochastic linear quadratic regulator problem, with integral quadratic constraints and indefinite control weights, is studied and under some conditions, the optimal control is described by solutions to an optimal problem and a stochastic Riccati equation with parameters. Our method (used to derive the norm optimal control) differs considerably from those developed in $[7,8]$. It seems that our method is more straightforward for our problem. Besides, the diffusion term of the system in our framework is allowed to be state-dependent. This makes our controlled system different from that in [8].

In this paper, we study the norm optimal control problem under suitable conditions on the controllability and the filtration. The rest of this paper is organized as follows. In Section 2, we consider the norm optimal control problem for approximately controllable systems with the natural filtration, and obtain necessary and sufficient conditions for its solvability. In Section 3, we study the same problem but for exactly controllable systems ("stronger" condition than that of Sect. 2) with a general filtration (weaker than the natural one). The main tools used in this section are the transposition solution to BSDEs and the Kalman-type condition guaranteeing the exact controllability.

We end this section by introducing the following notations:

- For any $t \in[0, T], L_{\mathcal{F}_{t}}^{2}\left(\Omega ; \mathbf{R}^{n}\right)$ is the Hilbert space of all $\mathcal{F}_{t^{-}}$-measurable, $\mathbf{R}^{n}$-valued random variables $\xi$ satisfying $\|\xi\|_{L_{\mathcal{F}_{t}}^{2}\left(\Omega ; \mathbf{R}^{n}\right)}^{2}=\mathbb{E}\|\xi\|^{2}<\infty$;

- $L_{\mathbb{F}}^{2}\left(\Omega ; D\left([0, T] ; \mathbf{R}^{n}\right)\right)$ is the Banach space of all $\mathbb{F}$-adapted, càdlàg stochastic processes $X$ satisfying $\|X\|_{L_{\mathbb{F}}^{2}\left(\Omega ; D\left([0, T] ; \mathbf{R}^{n}\right)\right)}^{2}=\mathbb{E}\left(\sup _{t \in[0, T]}\|X(t)\|^{2}\right)<\infty ;$

- $L_{\mathbb{F}}^{2}\left(\Omega ; C\left([0, T] ; \mathbf{R}^{n}\right)\right)$ is the subspace of $L_{\mathbb{F}}^{2}\left(\Omega ; D\left([0, T] ; \mathbf{R}^{n}\right)\right)$ consisting of all continuous processes;

- $L_{\mathbb{F}}^{2}\left((0, T) \times \Omega ; \mathbf{R}^{n}\right)$ is the Hilbert space of all $\mathbb{F}$-adapted stochastic processes $Y$ satisfying $\|Y\|_{L_{\mathbb{F}}^{2}\left((0, T) \times \Omega ; \mathbf{R}^{n}\right)}^{2}=$ $\left(\mathbb{E}\left(\int_{0}^{T}\|Y(t)\|^{2} \mathrm{~d} t\right)\right)<\infty$.

All of the above spaces are endowed with the canonical norms. Besides, we denote by $A^{*}$ the transport matrix of $A$; by $\langle\cdot, \cdot\rangle$ and $\|\cdot\|$ the usual inner products and norms in different Euclidean spaces respectively, which can be identified from the context. 


\section{Approximately Controllable systems With the NATURAl Filtration}

Consider the following stochastic linear control system with the natural filtration:

$$
\left\{\begin{aligned}
\mathrm{d} y(t) & =\left(A y(t)+A_{1} u_{1}(t)+B u_{2}(t)\right) \mathrm{d} t+\left(F_{1} y(t)+D u_{1}(t)\right) \mathrm{d} W(t), \quad t \in[0, T], \\
y(0) & =y_{0},
\end{aligned}\right.
$$

where $y(\cdot) \in L_{\mathbb{F}}^{2}\left(\Omega ; C\left([0, T] ; \mathbf{R}^{n}\right)\right), u_{1}(\cdot) \in L_{\mathbb{F}}^{2}\left((0, T) \times \Omega ; \mathbf{R}^{k}\right), u_{2}(\cdot) \in L_{\mathbb{F}}^{2}\left((0, T) \times \Omega ; \mathbf{R}^{l}\right)$ with $0 \leq k \leq n, l \geq 0$, $A \in \mathbf{R}^{n \times n}, A_{1} \in \mathbf{R}^{n \times k}, B \in \mathbf{R}^{n \times l}, F_{1} \in \mathbf{R}^{n \times n}, D \in \mathbf{R}^{n \times k}$ and $\operatorname{Rank} D=k$. (It can be easily verified that there exists a matrix $H \in \mathbf{R}^{n \times n}$ such that $H^{*} D+A_{1}=0$.) In the sequel, we denote by $\mathcal{H}_{a}$ the control space $L_{\mathbb{F}}^{2}\left((0, T) \times \Omega ; \mathbf{R}^{k}\right) \times L_{\mathbb{F}}^{2}\left((0, T) \times \Omega ; \mathbf{R}^{l}\right)$, endowed with the canonical norm

$$
\left\|\left(u_{1}, u_{2}\right)\right\|_{\mathcal{H}_{a}}=\left(\mathbb{E} \int_{0}^{T}\left(\left\|u_{1}(t)\right\|^{2}+\left\|u_{2}(t)\right\|^{2}\right) \mathrm{d} t\right)^{\frac{1}{2}}
$$

we denote by $y\left(\cdot ; u_{1}, u_{2}\right)$ the solution to the equation (2.1) corresponding to the control process $\left(u_{1}, u_{2}\right) \in \mathcal{H}_{a}$.

Definition 2.1. We say that the system (2.1) is approximately controllable (in the time interval $[0, T]$ ) if for any $y_{0} \in \mathbf{R}^{n}, y_{d} \in L_{\mathcal{F}_{T}}^{2}\left(\Omega, \mathbf{R}^{n}\right)$ and $r>0$, there exists a control $\left(u_{1}, u_{2}\right) \in \mathcal{H}_{a}$ such that the corresponding solution to (2.1) satisfies $\left\|y\left(T ; u_{1}, u_{2}\right)-y_{d}\right\|_{L_{\mathcal{F}_{T}}^{2}\left(\Omega ; \mathbf{R}^{n}\right)} \leq r$.

We quote the following known result (from [5], Prop. 5), which is a necessary and sufficient condition for the approximate controllability of the system (2.1).

Proposition 2.2. The system (2.1) (with the natural filtration) is approximately controllable if and only if any $(\varphi(\cdot), \psi(\cdot))$ solving the equation:

$$
\left\{\begin{aligned}
-\mathrm{d} \varphi(t) & =\left(\left(A^{*}+F_{1}^{*} H\right) \varphi(t)+F_{1}^{*} \psi(t)\right) \mathrm{d} t-(H \varphi(t)+\psi(t)) \mathrm{d} W(t), \quad t \in[0, T], \\
\varphi(T) & \in L_{\mathcal{F}_{T}}^{2}\left(\Omega ; \mathbf{R}^{n}\right),
\end{aligned}\right.
$$

and satisfying $B^{*} \varphi(t)=0$ and $D^{*} \psi(t)=0$, a.s., a.e. $t \in[0, T]$ is reduced trivially to 0 . Here $H$ is any matrix such that $H^{*} D+A_{1}=0$.

We refer to [10] for the well-posedness of the BSDE (2.2). Generally speaking, by the approximate controllability of the system, there exists at least one control steering the initial state to any neighborhood of a given state over some finite time horizon. It is natural to ask whether one could find a control of minimum energy to bring the initial state to the neighborhood of that given state. This is called the norm optimal control problem in the context of control theory (cf. e.g., $[4,13,17]$ and the references therein). In this section, we always assume that the system (2.1) is approximately controllable. For any $r>0, y_{0} \in \mathbf{R}^{n}$ and $y_{d} \in L_{\mathcal{F}_{T}}^{2}\left(\Omega ; \mathbf{R}^{n}\right)$, the norm optimal control problem (for (2.1)) is as follows:

$$
(\mathbf{N P})_{\mathbf{a}} \quad \inf _{\left(u_{1}, u_{2}\right) \in \mathcal{U}_{a}}\left\|\left(u_{1}, u_{2}\right)\right\|_{\mathcal{H}_{a}},
$$

where the admissible control set $\mathcal{U}_{a}$ is defined by

$$
\mathcal{U}_{a} \triangleq\left\{\left(u_{1}, u_{2}\right) \in \mathcal{H}_{a} ;\left\|y\left(T ; u_{1}, u_{2}\right)-y_{d}\right\|_{L_{\mathcal{F}_{T}}^{2}\left(\Omega ; \mathbf{R}^{n}\right)} \leq r\right\} .
$$

Notice that $\mathcal{U}_{a}$ is not empty because the system (2.1) is approximately controllable. We call $\left(\tilde{u}_{1}, \tilde{u}_{2}\right)$ a norm optimal control pair to the problem $(\mathbf{N P})_{\mathbf{a}}$ if

$$
\left\|\left(\tilde{u}_{1}, \tilde{u}_{2}\right)\right\|_{\mathcal{H}_{a}}=\inf _{\left(u_{1}, u_{2}\right) \in \mathcal{U}_{a}}\left\|\left(u_{1}, u_{2}\right)\right\|_{\mathcal{H}_{a}} .
$$


To present the main result of this section, we define a functional $J_{a}: L_{\mathcal{F}_{T}}^{2}\left(\Omega ; \mathbf{R}^{n}\right) \rightarrow \mathbf{R}$ by setting

$$
\begin{aligned}
J_{a}\left(\varphi_{T}\right)= & \frac{1}{2} \mathbb{E} \int_{0}^{T}\left(\left\|B^{*} \varphi(t)\right\|^{2}+\left\|D^{*} \psi(t)\right\|^{2}\right) \mathrm{d} t \\
& +r\left\|\varphi_{T}\right\|_{L_{\mathcal{F}_{T}}^{2}\left(\Omega ; \mathbf{R}^{n}\right)}+\mathbb{E}\left\langle\varphi(0), y_{0}\right\rangle-\mathbb{E}\left\langle\varphi_{T}, y_{d}\right\rangle
\end{aligned}
$$

where $(\varphi(\cdot), \psi(\cdot))$ solves the BSDE $(2.2)$ with $\varphi(T)=\varphi_{T} \in L_{\mathcal{F}_{T}}^{2}\left(\Omega ; \mathbf{R}^{n}\right)$. Now, the main result in this section is stated as follows:

Theorem 2.3. For the system (2.1), the following statements are equivalent:

(1) The system (2.1) is approximately controllable;

(2) For any $r>0, y_{0} \in \mathbf{R}^{n}$ and $y_{d} \in L_{\mathcal{F}_{T}}^{2}\left(\Omega ; \mathbf{R}^{n}\right)$, the functional $J_{a}(\cdot)$ admits a unique minimizer in $L_{\mathcal{F}_{T}}^{2}\left(\Omega ; \mathbf{R}^{n}\right)$

(3) For any $r>0, y_{0} \in \mathbf{R}^{n}$ and $y_{d} \in L_{\mathcal{F}_{T}}^{2}\left(\Omega ; \mathbf{R}^{n}\right)$, the problem $(\mathbf{N P})_{\mathbf{a}}$ has a unique optimal control.

Moreover, the minimizer $\hat{\varphi}_{T}$ of the functional $J_{a}(\cdot)$ over $L_{\mathcal{F}_{T}}^{2}\left(\Omega ; \mathbf{R}^{n}\right)$ reduces the unique norm optimal control pair $\left(\hat{u}_{1}, \hat{u}_{2}\right)$ to $(\mathbf{N P})_{\mathbf{a}}$ by

$$
\hat{u}_{1}(t)=D^{*} \hat{\psi}(t), \hat{u}_{2}(t)=B^{*} \hat{\varphi}(t), \text { for a.e. } t \in(0, T),
$$

where $(\hat{\varphi}(\cdot), \hat{\psi}(\cdot))$ solves the equation $(2.2)$ with the terminal condition $\varphi(T)=\hat{\varphi}_{T}$.

Remark 2.4. For the following general controlled system

$$
\left\{\begin{aligned}
\mathrm{d} y(t) & =(F y(t)+G u(t)) \mathrm{d} t+\left(F_{1} y(t)+G_{1} u(t)\right) \mathrm{d} W(t), \quad t \in[0, T], \\
y(0) & =y_{0},
\end{aligned}\right.
$$

we can transform it to the case (2.1). Indeed, suppose that Rank $G_{1}=k \leq \min \{m, n\}$. Then there exists an invertible matrix $M \in \mathbf{R}^{m \times m}$ such that $G_{1} M=(D, 0)$, where $D \in \mathbf{R}^{n \times k}$ satisfying Rank $D=k$. Set

$$
M^{-1} u=\left(\begin{array}{l}
u_{1} \\
u_{2}
\end{array}\right), G M=\left(A_{1}, B\right)
$$

where $\left(u_{1}(t), u_{2}(t)\right) \in \mathbf{R}^{k} \times \mathbf{R}^{m-k}$, for a.e. $t \in[0, T]$ and $A_{1} \in \mathbf{R}^{n \times k}, B \in \mathbf{R}^{n \times(m-k)}$. Therefore the system (2.5) is equivalent to (2.1) by setting $A=F$ and $l=m-k$.

If $\left(\hat{u}_{1}(\cdot), \hat{u}_{2}(\cdot)\right) \in \mathcal{H}_{a}$ and $\hat{y}(\cdot)$ are respectively the optimal control process and the optimal state process to the problem $(\mathbf{N P})_{\mathbf{a}}$ (for the system $\left.(2.1)\right)$, then $\hat{u}(\cdot)=M\left(\begin{array}{l}\hat{u}_{1}(\cdot) \\ \hat{u}_{2}(\cdot)\end{array}\right)$ and $\hat{y}(\cdot)$ are respectively the optimal control and state to the corresponding problem for the system $(2.5)$.

Before giving the proof of Theorem 2.3, we study some properties of the functional $J_{a}(\cdot)$ defined by $(2.3)$. We say a functional $L(\cdot)$ is coercive in $L_{\mathcal{F}_{T}}^{2}\left(\Omega ; \mathbf{R}^{n}\right)$ if

$$
L\left(\varphi_{T}^{m}\right) \rightarrow+\infty, \text { as } m \rightarrow+\infty,
$$

where $\left\{\varphi_{T}^{m}\right\}_{m \geq 1} \subset L_{\mathcal{F}_{T}}^{2}\left(\Omega ; \mathbf{R}^{n}\right)$ is any sequence such that

$$
\lim _{m \rightarrow \infty}\left\|\varphi_{T}^{m}\right\|_{L_{\mathcal{F}_{T}}^{2}\left(\Omega ; \mathbf{R}^{n}\right)}=+\infty .
$$

Lemma 2.5. Suppose that the system (2.1) is approximately controllable. Then the functional $J_{a}(\cdot)$ is coercive in $L_{\mathcal{F}_{T}}^{2}\left(\Omega ; \mathbf{R}^{n}\right)$. 
Proof. Let $\left\{\varphi_{T}^{m}\right\}_{m \geq 1} \subset L_{\mathcal{F}_{T}}^{2}\left(\Omega ; \mathbf{R}^{n}\right)$ be an arbitrary sequence such that

$$
\lim _{m \rightarrow \infty}\left\|\varphi_{T}^{m}\right\|_{L_{\mathcal{F}_{T}}^{2}\left(\Omega ; \mathbf{R}^{n}\right)}=+\infty
$$

Set

$$
\hat{\varphi}_{T}^{m}=\frac{\varphi_{T}^{m}}{\left\|\varphi_{T}^{m}\right\|_{L_{\mathcal{F}_{T}}^{2}\left(\Omega ; \mathbf{R}^{n}\right)}}, \text { for } m \in \mathbf{N}
$$

Clearly,

$$
\left\|\hat{\varphi}_{T}^{m}\right\|_{L_{\mathcal{F}_{T}}^{2}\left(\Omega ; \mathbf{R}^{n}\right)}=1, \quad m \geq 1 .
$$

By the definition $(2.3)$ of $J_{a}(\cdot)$, we have

$$
\frac{J_{a}\left(\varphi_{T}^{m}\right)}{\left\|\varphi_{T}^{m}\right\|_{L_{\mathcal{F}_{T}}^{2}\left(\Omega ; \mathbf{R}^{n}\right)}}=\frac{\left\|\varphi_{T}^{m}\right\|_{L_{\mathcal{F}_{T}}^{2}\left(\Omega ; \mathbf{R}^{n}\right)}}{2} \mathbb{E} \int_{0}^{T}\left(\left\|B^{*} \hat{\varphi}^{m}(t)\right\|^{2}+\left\|D^{*} \hat{\psi}^{m}(t)\right\|^{2}\right) \mathrm{d} t+r+\mathbb{E}\left\langle\hat{\varphi}^{m}(0), y_{0}\right\rangle-\mathbb{E}\left\langle\hat{\varphi}_{T}^{m}, y_{d}\right\rangle,
$$

where $\left(\hat{\varphi}^{m}, \hat{\psi}^{m}\right)$ solves the equation $(2.2)$ with the terminal condition $\varphi(T)=\hat{\varphi}_{T}^{m}$.

It suffices to prove that

$$
\varliminf_{m \rightarrow+\infty} \frac{J_{a}\left(\varphi_{T}^{m}\right)}{\left\|\varphi_{T}^{m}\right\|_{L_{\mathcal{F}_{T}}^{2}\left(\Omega ; \mathbf{R}^{n}\right)}} \geq r
$$

There are only two cases which are

$$
\varliminf_{m \rightarrow+\infty} \mathbb{E} \int_{0}^{T}\left(\left\|B^{*} \hat{\varphi}^{m}(t)\right\|^{2}+\left\|D^{*} \hat{\psi}^{m}(t)\right\|^{2}\right) \mathrm{d} t>0,
$$

and

$$
\varliminf_{m \rightarrow+\infty} \mathbb{E} \int_{0}^{T}\left(\left\|B^{*} \hat{\varphi}^{m}(t)\right\|^{2}+\left\|D^{*} \hat{\psi}^{m}(t)\right\|^{2}\right) \mathrm{d} t=0 .
$$

In the first case, (2.8) follows from (2.7) at once. In the second case, it follows from (2.6) that there are $\hat{\varphi}_{T}^{0} \in L_{\mathcal{F}_{T}}^{2}\left(\Omega ; \mathbf{R}^{n}\right)$ and a subsequence of $\left\{\hat{\varphi}_{T}^{m}\right\}_{m \geq 1}$, still denoted by itself, such that

$$
\hat{\varphi}_{T}^{m} \rightarrow \hat{\varphi}_{T}^{0} \text { weakly in } L_{\mathcal{F}_{T}}^{2}\left(\Omega ; \mathbf{R}^{n}\right) .
$$

We claim that

$$
\left(\hat{\varphi}^{m}(\cdot), \hat{\psi}^{m}(\cdot)\right) \rightarrow(\hat{\varphi}(\cdot), \hat{\psi}(\cdot)) \quad \text { weakly in } L_{\mathbb{F}}^{2}\left((0, T) \times \Omega ; \mathbf{R}^{n}\right) \times L_{\mathbb{F}}^{2}\left((0, T) \times \Omega ; \mathbf{R}^{n}\right),
$$

where $(\hat{\varphi}(\cdot), \hat{\psi}(\cdot))$ is the solution to the equation $(2.2)$ with $\varphi(T)=\hat{\varphi}_{T}^{0}$.

When (2.11) is proved, it follows from (2.9) and (2.11) that

$$
\mathbb{E} \int_{0}^{T}\left(\left\|B^{*} \hat{\varphi}(t)\right\|^{2}+\left\|D^{*} \hat{\psi}(t)\right\|^{2}\right) \mathrm{d} t=0 .
$$

Since the system (2.1) is approximately controllable, we derive from Proposition 2.2 that $\hat{\varphi}(t) \equiv 0$ for all $t \in(0, T)$. Then, (2.8) follows from (2.7) in this case.

The remainder is to show $(2.11)$. For any $(\xi(\cdot), \eta(\cdot)) \in L_{\mathbb{F}}^{2}\left((0, T) \times \Omega ; \mathbf{R}^{n}\right) \times L_{\mathbb{F}}^{2}\left((0, T) \times \Omega ; \mathbf{R}^{n}\right)$, let $S(\cdot)$ be the solution to the equation:

$$
\left\{\begin{aligned}
d S(t) & =(A S(t)+\xi(t)) \mathrm{d} t+\left(F_{1} S(t)+\eta(t)\right) \mathrm{d} W(t), \quad t \in[0, T] \\
S(0) & =0
\end{aligned}\right.
$$


By Itô's formula, we have that

$$
\mathbb{E}\left\langle S(T), \hat{\varphi}_{T}^{m}\right\rangle=\mathbb{E} \int_{0}^{T}\left\langle\xi(t), \hat{\varphi}^{m}(t)\right\rangle \mathrm{d} t+\mathbb{E} \int_{0}^{T}\left\langle\eta(t), H \hat{\varphi}^{m}(t)+\hat{\psi}^{m}(t)\right\rangle \mathrm{d} t .
$$

Similarly,

$$
\mathbb{E}\left\langle S(T), \hat{\varphi}_{T}^{0}\right\rangle=\mathbb{E} \int_{0}^{T}\langle\xi(t), \hat{\varphi}(t)\rangle \mathrm{d} t+\mathbb{E} \int_{0}^{T}\langle\eta(t), H \hat{\varphi}(t)+\hat{\psi}(t)\rangle \mathrm{d} t .
$$

These two equalities, as well as (2.10), imply that, as $m$ tends to $+\infty$,

$$
\mathbb{E} \int_{0}^{T}\left\langle\xi(t), \hat{\varphi}^{m}(t)\right\rangle \mathrm{d} t \rightarrow \mathbb{E} \int_{0}^{T}\langle\xi(t), \hat{\varphi}(t)\rangle \mathrm{d} t, \quad \forall \xi \in L_{\mathbb{F}}^{2}\left((0, T) \times \Omega ; \mathbf{R}^{n}\right)
$$

and

$$
\mathbb{E} \int_{0}^{T}\left\langle\eta(t), \hat{\psi}^{m}(t)\right\rangle \mathrm{d} t \rightarrow \mathbb{E} \int_{0}^{T}\langle\eta(t), \hat{\psi}(t)\rangle \mathrm{d} t, \quad \forall \eta \in L_{\mathbb{F}}^{2}\left((0, T) \times \Omega ; \mathbf{R}^{n}\right),
$$

which lead to (2.11).

Making use of Lemma 2.5, as well as Proposition 2.2, we can get the following properties of $J_{a}(\cdot)$.

Lemma 2.6. Suppose that the system (2.1) is approximately controllable. Then the functional $J_{a}(\cdot)$ is strictly convex. Consequently, $J_{a}(\cdot)$ admits a unique minimizer $\hat{\varphi}_{T}$ in $L_{\mathcal{F}_{T}}^{2}\left(\Omega ; \mathbf{R}^{n}\right)$. Furthermore, $\hat{\varphi}_{T}=0$ if and only if $\left\|y(T ; 0,0)-y_{d}\right\|_{L_{\mathcal{F}_{T}}^{2}\left(\Omega ; \mathbf{R}^{n}\right)} \leq r$.

Proof. We first show the strict convexity of $J_{a}(\cdot)$, i.e., for any $\alpha \in(0,1)$,

$$
J_{a}\left(\alpha \varphi_{T}+(1-\alpha) \bar{\varphi}_{T}\right)<\alpha J_{a}\left(\varphi_{T}\right)+(1-\alpha) J_{a}\left(\bar{\varphi}_{T}\right) \text {, for any } \varphi_{T} \neq \bar{\varphi}_{T} \in L_{\mathcal{F}_{T}}^{2}\left(\Omega ; \mathbf{R}^{n}\right) .
$$

Without loss of generality, we can assume that $\varphi_{T} \neq 0$. It follows from $(2.3)$ that

$$
\begin{aligned}
& J_{a}\left(\alpha \varphi_{T}+(1-\alpha) \bar{\varphi}_{T}\right) \\
= & \alpha J_{a}\left(\varphi_{T}\right)+(1-\alpha) J_{a}\left(\bar{\varphi}_{T}\right) \\
& +r\left\|\alpha \varphi_{T}+(1-\alpha) \bar{\varphi}_{T}\right\|_{L_{\mathcal{F}_{T}}^{2}\left(\Omega ; \mathbf{R}^{n}\right)}-r\left(\left\|\alpha \varphi_{T}\right\|_{L_{\mathcal{F}_{T}}^{2}\left(\Omega ; \mathbf{R}^{n}\right)}+(1-\alpha)\left\|\bar{\varphi}_{T}\right\|_{L_{\mathcal{F}_{T}}^{2}\left(\Omega ; \mathbf{R}^{n}\right)}\right) \\
& +\frac{\alpha(\alpha-1)}{2} \mathbb{E} \int_{0}^{T}\left(\left\|B^{*} \varphi(t)-B^{*} \bar{\varphi}(t)\right\|^{2}+\left\|D^{*} \psi(t)-D^{*} \bar{\psi}(t)\right\|^{2}\right) \mathrm{d} t .
\end{aligned}
$$

Now, if

$$
\left\|\alpha \varphi_{T}+(1-\alpha) \bar{\varphi}_{T}\right\|_{L_{\mathcal{F}_{T}}^{2}\left(\Omega ; \mathbf{R}^{n}\right)}<\alpha\left\|\varphi_{T}\right\|_{L_{\mathcal{F}_{T}}^{2}\left(\Omega ; \mathbf{R}^{n}\right)}+(1-\alpha)\left\|\bar{\varphi}_{T}\right\|_{L_{\mathcal{F}_{T}}^{2}\left(\Omega ; \mathbf{R}^{n}\right)},
$$

then (2.12) is obviously valid from (2.13). On the other hand, if

$$
\left\|\alpha \varphi_{T}+(1-\alpha) \bar{\varphi}_{T}\right\|_{L_{\mathcal{F}_{T}}^{2}\left(\Omega ; \mathbf{R}^{n}\right)}=\alpha\left\|\varphi_{T}\right\|_{L_{\mathcal{F}_{T}}^{2}\left(\Omega ; \mathbf{R}^{n}\right)}+(1-\alpha)\left\|\bar{\varphi}_{T}\right\|_{L_{\mathcal{F}_{T}}^{2}\left(\Omega ; \mathbf{R}^{n}\right)},
$$

then $\bar{\varphi}_{T}=k \varphi_{T}$ for some $k>0$ with $k \neq 1$. From the uniqueness of the solution to the equation (2.2), we have that

$$
\bar{\varphi}(t)=k \varphi(t), \bar{\psi}(t)=k \psi(t), \text { a.e. } t \in(0, T) .
$$

Hence,

$$
\mathbb{E} \int_{0}^{T}\left(\left\|B^{*} \varphi(t)-B^{*} \bar{\varphi}(t)\right\|^{2}+\left\|D^{*} \psi(t)-D^{*} \bar{\psi}(t)\right\|^{2}\right) \mathrm{d} t=(1-k)^{2} \mathbb{E} \int_{0}^{T}\left(\left\|B^{*} \varphi(t)\right\|^{2}+\left\|D^{*} \psi(t)\right\|^{2}\right) \mathrm{d} t .
$$


Since the system (2.1) is approximately controllable and $\varphi_{T} \neq 0$, it follows from Proposition 2.2 that

$$
\mathbb{E} \int_{0}^{T}\left(\left\|B^{*} \varphi(t)\right\|^{2}+\left\|D^{*} \psi(t)\right\|^{2}\right) \mathrm{d} t>0,
$$

from which, as well as (2.13) and (2.14), the desired inequality (2.12) follows.

We next show the existence and uniqueness of the minimizer of $J_{a}(\cdot)$ in $L_{\mathcal{F}_{T}}^{2}\left(\Omega ; \mathbf{R}^{n}\right)$. Let $d=$ $\inf _{\varphi_{T} \in L_{\mathcal{F}_{T}}^{2}\left(\Omega ; \mathbf{R}^{n}\right)} J_{a}\left(\varphi_{T}\right)$. By Lemma 2.5, we have that $J_{a}(\cdot)$ is coercive, and then $d>-\infty$. Now, there exists a sequence $\left\{\varphi_{T}^{m}\right\}_{m \geq 1} \subset L_{\mathcal{F}_{T}}^{2}\left(\Omega ; \mathbf{R}^{n}\right)$, such that $d \leq J_{a}\left(\varphi_{T}^{m}\right)<d+\frac{1}{m}$. By the coercivity of $J_{a}(\cdot)$, we derive that $\left\|\varphi_{T}^{m}\right\|_{L_{\mathcal{F}_{T}}^{2}\left(\Omega ; \mathbf{R}^{n}\right)} \leq M$ for some $M>0$. Hence there exists a subsequence of $\left\{\varphi_{T}^{m}\right\}_{m \geq 1}$ converging weakly to $\hat{\varphi}_{T}$ in $L_{\mathcal{F}_{T}}^{2}\left(\Omega ; \mathbf{R}^{n}\right)$. We still use $\left\{\varphi_{T}^{m}\right\}_{m \geq 1}$ to denote this subsequence. By the same way to show (2.11) (in the proof of Lemma 2.5), we can verify that

$$
\left(\varphi^{m}, \psi^{m}\right) \rightarrow(\hat{\varphi}, \hat{\psi}) \quad \text { weakly in } L_{\mathbb{F}}^{2}\left((0, T) \times \Omega ; \mathbf{R}^{n}\right) \times L_{\mathbb{F}}^{2}\left((0, T) \times \Omega ; \mathbf{R}^{n}\right) .
$$

Since $J_{a}(\cdot)$ is weakly semi-continuous, it holds that

$$
d \leq J_{a}\left(\hat{\varphi}_{T}\right) \leq \varliminf_{m \rightarrow \infty} J_{a}\left(\varphi_{T}^{m}\right) \leq \underline{\underline{\lim }}\left(d+\frac{1}{m}\right)=d .
$$

Hence $\hat{\varphi}_{T}$ is a minimizer of $J_{a}(\cdot)$. The uniqueness of the minimizer of $J_{a}(\cdot)$ follows from the strictly convexity of $J_{a}(\cdot)$.

Finally, we show that

$$
\hat{\varphi}_{T}=0 \Leftrightarrow\left\|y(T ; 0,0)-y_{d}\right\|_{L_{\mathcal{F}_{T}}^{2}\left(\Omega ; \mathbf{R}^{n}\right)} \leq r .
$$

Suppose that $\hat{\varphi}_{T}=0$. Then it follows from (2.3) that for each $\varphi_{T} \in L_{\mathcal{F}_{T}}^{2}\left(\Omega ; \mathbf{R}^{n}\right)$,

$$
0 \leq \lim _{h \rightarrow 0^{+}} \frac{J_{a}\left(\hat{\varphi}_{T}+h \varphi_{T}\right)-J_{a}\left(\hat{\varphi}_{T}\right)}{h}=r\left\|\varphi_{T}\right\|_{L_{\mathcal{F}_{T}}^{2}\left(\Omega ; \mathbf{R}^{n}\right)}+\mathbb{E}\left\langle\varphi(0), y_{0}\right\rangle-\mathbb{E}\left\langle\varphi_{T}, y_{d}\right\rangle .
$$

By Itô's formula, we see that

$$
\mathbb{E}\left\langle\varphi(0), y_{0}\right\rangle-\mathbb{E}\left\langle\varphi_{T}, y(T ; 0,0)\right\rangle=0, \quad \forall \varphi_{T} \in L_{\mathcal{F}_{T}}^{2}\left(\Omega ; \mathbf{R}^{n}\right) .
$$

Hence,

$$
\mathbb{E}\left\langle\varphi_{T}, y_{d}-y(T ; 0,0)\right\rangle \leq r\left\|\varphi_{T}\right\|_{L_{\mathcal{F}_{T}}^{2}\left(\Omega ; \mathbf{R}^{n}\right)}, \quad \forall \varphi_{T} \in L_{\mathcal{F}_{T}}^{2}\left(\Omega ; \mathbf{R}^{n}\right),
$$

which leads to $\left\|y(T ; 0,0)-y_{d}\right\|_{L_{\mathcal{F}_{T}}^{2}\left(\Omega ; \mathbf{R}^{n}\right)} \leq r$.

Conversely, we suppose that $\left\|y(T ; 0,0)-y_{d}\right\|_{L_{\mathcal{F}_{T}}^{2}\left(\Omega ; \mathbf{R}^{n}\right)} \leq r$. Then for any $\varphi_{T} \in L_{\mathcal{F}_{T}}^{2}\left(\Omega ; \mathbf{R}^{n}\right)$,

$$
\begin{aligned}
J_{a}\left(\varphi_{T}\right) & \geq r\left\|\varphi_{T}\right\|_{L_{\mathcal{F}_{T}}^{2}\left(\Omega ; \mathbf{R}^{n}\right)}+\mathbb{E}\left\langle\varphi(0), y_{0}\right\rangle-\mathbb{E}\left\langle\varphi_{T}, y_{d}\right\rangle \\
& =r\left\|\varphi_{T}\right\|_{L_{\mathcal{F}_{T}}^{2}\left(\Omega ; \mathbf{R}^{n}\right)}+\mathbb{E}\left\langle\varphi_{T}, y(T ; 0,0)-y_{d}\right\rangle \\
& \geq r\left\|\varphi_{T}\right\|_{L_{\mathcal{F}_{T}}^{2}\left(\Omega ; \mathbf{R}^{n}\right)}-\left\|\varphi_{T}\right\|_{L_{\mathcal{F}_{T}}^{2}\left(\Omega ; \mathbf{R}^{n}\right)}\left\|y(T ; 0,0)-y_{d}\right\|_{L_{\mathcal{F}_{T}}^{2}\left(\Omega ; \mathbf{R}^{n}\right)} \\
& \geq 0=J_{a}(0)
\end{aligned}
$$

which leads to $\hat{\varphi}_{T}=0$. This completes the proof.

We are now in a position to prove Theorem 2.3. 
Proof of Theorem 2.3.

(1) $\Rightarrow(2)$. It is clear from Lemma 2.6.

$(3) \Rightarrow(1)$. Since for any $r>0, y_{0} \in \mathbf{R}^{n}$ and $y_{d} \in L_{\mathcal{F}_{T}}^{2}\left(\Omega ; \mathbf{R}^{n}\right)$, the problem $(\mathbf{N P})_{\mathbf{a}}$ admits an optimal control pair, then the system (2.1) is approximately controllable.

$(2) \Rightarrow(3)$. Let $\hat{\varphi}_{T}$ be the minimizer of $J_{a}(\cdot)$. Then, the Euler-Lagrange equation associated to $J_{a}(\cdot)$ reads as follows:

$$
\begin{aligned}
& \mathbb{E} \int_{0}^{T}\left(\left\langle B^{*} \hat{\varphi}(t), B^{*} \varphi(t)\right\rangle+\left\langle D^{*} \hat{\psi}(t), D^{*} \psi(t)\right\rangle\right) \mathrm{d} t \\
& +\mathbb{E}\left\langle\varphi(0), y_{0}\right\rangle+r \mathbb{E}\left\langle\frac{\hat{\varphi}_{T}}{\left\|\hat{\varphi}_{T}\right\|_{L_{\mathcal{F}_{T}}^{2}\left(\Omega ; \mathbf{R}^{n}\right)}}, \varphi_{T}\right\rangle-\mathbb{E}\left\langle\varphi_{T}, y_{d}\right\rangle=0, \quad \forall \varphi_{T} \in L_{\mathcal{F}_{T}}^{2}\left(\Omega ; \mathbf{R}^{n}\right) .
\end{aligned}
$$

By Itô's formula, we see that for each $\varphi_{T} \in L_{\mathcal{F}_{T}}^{2}\left(\Omega ; \mathbf{R}^{n}\right)$,

$$
\mathbb{E}\left\langle\varphi_{T}, y\left(T ; u_{1}, u_{2}\right)\right\rangle-\mathbb{E}\left\langle\varphi(0), y_{0}\right\rangle=\mathbb{E} \int_{0}^{T}\left(\left\langle u_{2}(t), B^{*} \varphi(t)\right\rangle+\left\langle u_{1}(t), D^{*} \psi(t)\right\rangle\right) \mathrm{d} t .
$$

Let $\left(\hat{u}_{1}, \hat{u}_{2}\right)$ be given by (2.4). We first claim that it is an admissible control pair to the problem $(\mathbf{N P})_{\mathbf{a}}$. Indeed, it follows from (2.15), (2.16) and (2.4) that

$$
\mathbb{E}\left\langle y\left(T ; \hat{u}_{1}, \hat{u}_{2}\right)-y_{d}+r \frac{\hat{\varphi}_{T}}{\left\|\hat{\varphi}_{T}\right\|_{L_{\mathcal{F}_{T}}^{2}\left(\Omega ; \mathbf{R}^{n}\right)}}, \varphi_{T}\right\rangle=0, \quad \forall \varphi_{T} \in L_{\mathcal{F}_{T}}^{2}\left(\Omega ; \mathbf{R}^{n}\right) .
$$

That is

$$
y\left(T ; \hat{u}_{1}, \hat{u}_{2}\right)=y_{d}-r \frac{\hat{\varphi}_{T}}{\left\|\hat{\varphi}_{T}\right\|_{L_{\mathcal{F}_{T}}^{2}\left(\Omega ; \mathbf{R}^{n}\right)}} .
$$

Hence, $\left(\hat{u}_{1}, \hat{u}_{2}\right)$ is admissible to $(\mathbf{N P})_{\mathbf{a}}$.

We then claim that $\left(\hat{u}_{1}, \hat{u}_{2}\right)$ is the unique optimal control to the problem $(\mathbf{N P})_{\mathbf{a}}$. To this end, let $\left(u_{1}, u_{2}\right)$ be any admissible control pair to $(\mathbf{N P})_{\mathbf{a}}$. Consequently,

$$
\mathbb{E}\left\langle y\left(T ; u_{1}, u_{2}\right), \varphi_{T}\right\rangle \geq-r\left\|\varphi_{T}\right\|_{L_{\mathcal{F}_{T}}^{2}\left(\Omega ; \mathbf{R}^{n}\right)}+\mathbb{E}\left\langle y_{d}, \varphi_{T}\right\rangle, \quad \forall \varphi_{T} \in L_{\mathcal{F}_{T}}^{2}\left(\Omega ; \mathbf{R}^{n}\right) .
$$

By letting $\varphi_{T}=\hat{\varphi}_{T}$ in both (2.15) and (2.16), we obtain from (2.4), as well as (2.17), that

$$
\begin{aligned}
& \mathbb{E} \int_{0}^{T}\left(\left\|\hat{u}_{1}(t)\right\|^{2}+\left\|\hat{u}_{2}(t)\right\|^{2}\right) \mathrm{d} t=\mathbb{E} \int_{0}^{T}\left(\left\|B^{*} \hat{\varphi}(t)\right\|^{2}+\left\|D^{*} \hat{\psi}(t)\right\|^{2}\right) \mathrm{d} t \\
= & -\mathbb{E}\left\langle\hat{\varphi}(0), y_{0}\right\rangle-r\left\|\hat{\varphi}_{T}\right\|_{L_{\mathcal{F}_{T}}^{2}\left(\Omega ; \mathbf{R}^{n}\right)}+\mathbb{E}\left\langle\hat{\varphi}_{T}, y_{d}\right\rangle \\
\leq & \mathbb{E}\left\langle\hat{\varphi}_{T}, y\left(T ; u_{1}, u_{2}\right)\right\rangle-\mathbb{E}\left\langle\hat{\varphi}(0), y_{0}\right\rangle=\mathbb{E} \int_{0}^{T}\left(\left\langle u_{1}(t), \hat{u}_{1}(t)\right\rangle+\left\langle u_{2}(t), \hat{u}_{2}(t)\right\rangle\right) \mathrm{d} t .
\end{aligned}
$$

Hence,

$$
\mathbb{E} \int_{0}^{T}\left(\left\|\hat{u}_{1}(t)\right\|^{2}+\left\|\hat{u}_{2}(t)\right\|^{2}\right) \mathrm{d} t \leq \mathbb{E} \int_{0}^{T}\left(\left\langle u_{1}(t), \hat{u}_{1}(t)\right\rangle+\left\langle u_{2}(t), \hat{u}_{2}(t)\right\rangle\right) \mathrm{d} t .
$$

This, together with the Cauchy-Schwartz inequality, implies the optimality of the control pair $\left(\hat{u}_{1}, \hat{u}_{2}\right)$. The uniqueness of the optimal control pair to $(\mathbf{N P})_{\mathbf{a}}$ follows immediately from the classical parallelogram rule of the norm $\|\cdot\|_{\mathcal{H}_{a}}$. Hence, the proof is completed. 
Remark 2.7. If $F_{1}=0, k=0$, then $A_{1}=D=0$, hence the system (2.1) degenerates to

$$
\left\{\begin{aligned}
\mathrm{d} y(t) & =\left(A y(t)+B u_{2}(t)\right) \mathrm{d} t, \quad t \in[0, T], \\
y(0) & =y_{0} .
\end{aligned}\right.
$$

Meanwhile, the dual equation (2.2) degenerates to

$$
\left\{\begin{aligned}
-\mathrm{d} \varphi(t) & =A^{*} \varphi(t) \mathrm{d} t-\psi(t) \mathrm{d} W(t), \quad t \in[0, T], \\
\varphi(T) & \in L_{\mathcal{F}_{T}}^{2}\left(\Omega ; \mathbf{R}^{n}\right) .
\end{aligned}\right.
$$

$(\mathbf{N P})_{\mathbf{a}}$ and $J_{a}(\cdot)$ turn to respectively

$$
(\mathbf{N P})_{\mathbf{a}}^{*} \quad \inf _{u_{2} \in \mathcal{U}_{a}}\left\|u_{2}\right\|_{L_{\mathbb{F}}^{2}\left((0, T) \times \Omega ; \mathbf{R}^{l}\right)}
$$

and

$$
J_{a}^{*}\left(\varphi_{T}\right)=\frac{1}{2} \mathbb{E} \int_{0}^{T}\left\|B^{*} \varphi(t)\right\|^{2} \mathrm{~d} t+r\left\|\varphi_{T}\right\|_{L_{\mathcal{F}_{T}}^{2}\left(\Omega ; \mathbf{R}^{n}\right)}+\mathbb{E}\left\langle\varphi(0), y_{0}\right\rangle-\mathbb{E}\left\langle\varphi_{T}, y_{d}\right\rangle
$$

From Theorem 2.3, $J_{a}^{*}(\cdot)$ admits a unique minimizer $\hat{\varphi}_{T} \in L_{\mathcal{F}_{T}}^{2}\left(\Omega ; \mathbf{R}^{n}\right)$, and the unique optimal control to $(\mathbf{N P})_{\mathbf{a}}^{*}$ is given by $\hat{u}_{2}(\cdot)=B^{*} \hat{\varphi}(\cdot) \in L_{\mathbb{F}}^{2}\left((0, T) \times \Omega ; \mathbf{R}^{l}\right)$.

We now view the system $(2.18)$ as a deterministic control system

$$
\left\{\begin{array}{l}
\dot{\bar{y}}(t)=A \bar{y}(t)+B \bar{u}_{2}(t), \quad t \in[0, T] \\
\bar{y}(0)=y_{0}
\end{array}\right.
$$

with $\bar{u}_{2}(\cdot) \in L^{2}\left(0, T ; \mathbf{R}^{l}\right)$. Since the stochastic system (2.18) is approximately controllable, the deterministic one (2.21) is approximately/exactly controllable as well. Hence for any $r>0$ and $\bar{y}_{d} \in \mathbf{R}^{n}$, we can also consider the norm control problem

$$
(\mathbf{N P})_{\mathbf{a}}^{* *} \quad \inf _{u_{2} \in \mathcal{U}_{a}^{*}}\left\|u_{2}\right\|_{L^{2}\left(0, T ; \mathbf{R}^{l}\right)}
$$

with

$$
\mathcal{U}_{a}^{*} \triangleq\left\{u_{2}(\cdot) \in L^{2}\left(0, T ; \mathbf{R}^{l}\right) ;\left\|\bar{y}\left(T ; u_{2}\right)-\bar{y}_{d}\right\| \leq r\right\} .
$$

Define a functional $J_{a}^{* *}: \mathbf{R}^{n} \rightarrow \mathbf{R}$ by setting

$$
J_{a}^{* *}\left(\bar{\varphi}_{T}\right)=\frac{1}{2} \int_{0}^{T}\left\|B^{*} \bar{\varphi}(t)\right\|^{2} \mathrm{~d} t+r\left\|\bar{\varphi}_{T}\right\|+\left\langle\bar{\varphi}(0), y_{0}\right\rangle-\left\langle\bar{\varphi}_{T}, \bar{y}_{d}\right\rangle
$$

where $\varphi(\cdot)$ solves the following deterministic equation:

$$
\left\{\begin{aligned}
-\dot{\bar{\varphi}}(t) & =A^{*} \bar{\varphi}(t), \quad t \in[0, T] \\
\bar{\varphi}(T) & =\bar{\varphi}_{T}
\end{aligned}\right.
$$

By a similar method as that to prove Theorem 2.3, we can show that $J_{a}^{* *}(\cdot)$ admits a unique minimizer $\hat{\bar{\varphi}}_{T} \in \mathbf{R}^{n}$, and the unique optimal control to $(\mathbf{N P})_{\mathbf{a}}^{* *}$ is $\hat{\bar{u}}_{2}(\cdot)=B^{*} \hat{\bar{\varphi}}(\cdot) \in L^{2}\left(0, T ; \mathbf{R}^{l}\right)$.

In the above two problems (NP) $)_{\mathbf{a}}^{*}$ and $(\mathbf{N P})_{\mathbf{a}}^{* *}$, if we take $y_{d}=\bar{y}_{d} \in \mathbf{R}^{n}$, then

$$
\hat{\varphi}_{T}=\hat{\bar{\varphi}}_{T}, \hat{u}_{2}(\cdot)=\hat{\bar{u}}_{2}(\cdot) \text {. }
$$

In fact, it is obvious to get the second equality if we have $\hat{\varphi}_{T}=\hat{\bar{\varphi}}_{T}$, hence we need only to prove the first one. It is easy to see that $(\mathbb{E} \hat{\varphi}(\cdot), 0)$ solves the equation $(2.19)$ with $\varphi(T)=\mathbb{E} \hat{\varphi}_{T}$. Notice that for each random 
variable $\xi$, it holds that $\mathbb{E}\|\xi\|^{2} \geq\|\mathbb{E} \xi\|^{2}$. Then, we see that

$$
\begin{aligned}
J_{a}^{*}\left(\mathbb{E} \hat{\varphi}_{T}\right) & =\frac{1}{2} \mathbb{E} \int_{0}^{T}\left\|B^{*}(\mathbb{E} \hat{\varphi}(t))\right\|^{2} \mathrm{~d} t+r\left\|\mathbb{E} \hat{\varphi}_{T}\right\|_{L_{\mathcal{F}_{T}}^{2}\left(\Omega ; \mathbf{R}^{n}\right)}+\mathbb{E}\left\langle\mathbb{E} \hat{\varphi}(0), y_{0}\right\rangle-\mathbb{E}\left\langle\mathbb{E} \hat{\varphi}_{T}, y_{d}\right\rangle \\
& \leq \frac{1}{2} \mathbb{E} \int_{0}^{T}\left\|B^{*} \hat{\varphi}(t)\right\|^{2} \mathrm{~d} t+r\left\|\hat{\varphi}_{T}\right\|_{L_{\mathcal{F}_{T}}^{2}\left(\Omega ; \mathbf{R}^{n}\right)}+\mathbb{E}\left\langle\hat{\varphi}(0), y_{0}\right\rangle-\mathbb{E}\left\langle\hat{\varphi}_{T}, y_{d}\right\rangle \\
& =J_{a}^{*}\left(\hat{\varphi}_{T}\right) .
\end{aligned}
$$

Hence, $\hat{\varphi}_{T}=\mathbb{E} \hat{\varphi}_{T}$. Furthermore, using $y_{d}=\bar{y}_{d}$ and the uniquely minimal property of $\hat{\bar{\varphi}}_{T}$ for $J_{a}^{* *}(\cdot)$, we have

$$
J_{a}^{*}\left(\hat{\varphi}_{T}\right)=J_{a}^{* *}\left(\hat{\varphi}_{T}\right) \geq J_{a}^{* *}\left(\hat{\bar{\varphi}}_{T}\right)=J_{a}^{*}\left(\hat{\bar{\varphi}}_{T}\right) \geq J_{a}^{*}\left(\hat{\varphi}_{T}\right)
$$

which yields that

$$
\hat{\varphi}_{T}=\hat{\bar{\varphi}}_{T}
$$

Remark 2.8. Now we consider the norm optimal control problem for the following system driving by a 1dimensional Brownian motion $\{W(t) ; t \geq 0\}$ and a Poisson random measure $N$ on $\mathbf{R}^{+} \times E(E=\mathbf{R} \backslash\{0\})$ defined on $\left(\Omega, \mathcal{F}, \mathcal{F}_{t}^{W, N}, P ; t \geq 0\right)$ :

$$
\left\{\begin{aligned}
\mathrm{d} y(t) & =(A y(t)+B u(t)) \mathrm{d} t+C y(t) \mathrm{d} W(t)+\int_{E} D(z) y(t) \tilde{N}(\mathrm{~d} t, \mathrm{~d} z), \quad t \in(0, T), \\
y(0) & =y_{0} .
\end{aligned}\right.
$$

Here $y(\cdot) \in L_{\mathbb{F}}^{2}\left(\Omega ; D\left([0, T] ; \mathbf{R}^{n}\right)\right), u(\cdot) \in L_{\mathbb{F}}^{2}\left((0, T) \times \Omega ; \mathbf{R}^{m}\right), A \in \mathbf{R}^{n \times n}, B \in \mathbf{R}^{n \times m}, C \in \mathbf{R}^{n \times n}$ and $D: E \rightarrow$ $\mathbf{R}^{n \times n}$ is $\mathcal{B}(E)$-measurable satisfying $\int_{E}\|D(z)\|^{2} \lambda(\mathrm{d} z)<\infty$, where $\lambda$ is the intensity (Lévy measure) of $N$ with the property that

$$
\int_{E}\left(1 \wedge|z|^{2}\right) \lambda(\mathrm{d} z)<\infty
$$

In this case, the norm optimal control problem reads as follows: Fixed $T>0, r>0, y_{0} \in \mathbf{R}^{n}$ and $y_{d} \in$ $L_{\mathcal{F}_{T}}^{2}\left(\Omega ; \mathbf{R}^{n}\right)$, consider

$$
(\mathbf{N P})_{\mathbf{a}}^{\prime} \quad \inf _{u \in \mathcal{U}_{a}^{\prime}}\|u\|_{L_{\mathbb{F}}^{2}\left((0, T) \times \Omega ; \mathbf{R}^{m}\right)},
$$

where the admissible control set $\mathcal{U}_{a}^{\prime}$ is defined by

$$
\mathcal{U}_{a}^{\prime} \triangleq\left\{u \in L_{\mathbb{F}}^{2}\left((0, T) \times \Omega ; \mathbf{R}^{m}\right) ;\left\|y(T ; u)-y_{d}\right\|_{L_{\mathcal{F}_{T}}^{2}\left(\Omega ; \mathbf{R}^{n}\right)} \leq r\right\} .
$$

Also, we define for each $\varphi_{T} \in L_{\mathcal{F}_{T}}^{2}\left(\Omega ; \mathbf{R}^{n}\right)$, a functional $J_{a}^{\prime}: L_{\mathcal{F}_{T}}^{2}\left(\Omega ; \mathbf{R}^{n}\right) \rightarrow \mathbf{R}$ by setting

$$
J_{a}^{\prime}\left(\varphi_{T}\right)=\frac{1}{2} \mathbb{E} \int_{0}^{T}\left\|B^{*} \varphi(t)\right\|^{2} \mathrm{~d} t+r\left\|\varphi_{T}\right\|_{L_{\mathcal{F}_{T}}^{2}\left(\Omega ; \mathbf{R}^{n}\right)}+\mathbb{E}\left\langle\varphi(0), y_{0}\right\rangle-\mathbb{E}\left\langle\varphi_{T}, y_{d}\right\rangle,
$$

where $(\varphi(\cdot), \psi(\cdot), \phi(\cdot))$ solves the following BSDE with jump

$$
\left\{\begin{aligned}
-\mathrm{d} \varphi(t) & =\left(A^{*} \varphi(t)+C^{*} \psi(t)+\int_{E} D^{*}(z) \lambda(\mathrm{d} z)\right) \mathrm{d} t-\psi(t) \mathrm{d} W(t)-\int_{E} \phi(z, t) \tilde{N}(\mathrm{~d} t \mathrm{~d} z), \\
\varphi(T) & =\varphi_{T} .
\end{aligned}\right.
$$


We refer to [12] for the well-posedness of the equation (2.25). Following the same arguments as those in the proof of Theorem 2.3, we can obtain the following result:

Theorem 2.9. The following statements are equivalent:

(1) The system (2.24) is approximately controllable;

(2) For any $r>0, y_{0} \in \mathbf{R}^{n}$ and $y_{d} \in L_{\mathcal{F}_{T}}^{2}\left(\Omega ; \mathbf{R}^{n}\right)$, the functional $J_{a}^{\prime}(\cdot)$ admits a unique minimizer in $L_{\mathcal{F}_{T}}^{2}\left(\Omega ; \mathbf{R}^{n}\right)$

(3) For any $r>0, y_{0} \in \mathbf{R}^{n}$ and $y_{d} \in L_{\mathcal{F}_{T}}^{2}\left(\Omega ; \mathbf{R}^{n}\right)$, the norm control problem $(\mathbf{N P})_{\mathbf{a}}^{\prime}$ has a unique optimal control.

Moreover, if $\hat{\varphi}_{T}$ is the minimizer of the functional $J_{a}^{\prime}(\cdot)$ in $L_{\mathcal{F}_{T}}^{2}\left(\Omega ; \mathbf{R}^{n}\right)$, then $\hat{u}$ defined by

$$
\hat{u}(t)=B^{*} \hat{\varphi}(t) \text {, as, a.e. } t \in(0, T),
$$

is the unique norm optimal control to the problem $(\mathbf{N P})_{\mathbf{a}}^{\prime}$, where $\hat{\varphi}(\cdot)$ solves the equation $(2.25)$ with the terminal condition $\varphi(T)=\hat{\varphi}_{T}$.

\section{ExaCtly CONTROLlable Systems With A GENERAL FiLtration}

In this section, we do not assume that $(\Omega, \mathcal{F}, \mathbb{F}, P)$ is the natural filtration space. Let us consider the following exactly controllable stochastic linear control system:

$$
\left\{\begin{aligned}
\mathrm{d} y(t) & =\left(A y(t)+A_{1} u_{1}(t)+B u_{2}(t)\right) \mathrm{d} t+u_{1}(t) \mathrm{d} W(t), \quad t \in[0, T], \\
y(0) & =y_{0},
\end{aligned}\right.
$$

where $u_{1}(\cdot) \in L_{\mathbb{F}}^{2}\left((0, T) \times \Omega ; \mathbf{R}^{n}\right)$ and $u_{2}(\cdot) \in L_{\mathbb{F}}^{2}\left((0, T) \times \Omega ; \mathbf{R}^{l}\right)$ are controls, and $A \in \mathbf{R}^{n \times n}, A_{1} \in \mathbf{R}^{n \times n}, B \in$ $\mathbf{R}^{n \times l}$. For simplicity, let $\mathcal{H}$ denote the space $L_{\mathbb{F}}^{2}\left((0, T) \times \Omega ; \mathbf{R}^{n}\right) \times L_{\mathbb{F}}^{2}\left((0, T) \times \Omega ; \mathbf{R}^{l}\right)$ endowed with the canonical norm.

Since $(\Omega, \mathcal{F}, \mathbb{F}, P)$ is a general complete filtration space, similar to [9], we define the transposition solution (which is different from the strong solution defined by Pardoux and Peng [10]) to BSDE, and then give the definition of exact controllability of the system (3.1).

Let us assume that $f: \Omega \times[0, T] \times \mathbf{R}^{n} \times \mathbf{R}^{n} \rightarrow \mathbf{R}^{n}$ is measurable with respect to $\mathcal{P} \otimes \mathcal{B}\left(\mathbf{R}^{n}\right) \otimes \mathcal{B}\left(\mathbf{R}^{n}\right) / \mathcal{B}\left(\mathbf{R}^{n}\right)$, and $g: \mathbf{R}^{n} \rightarrow \mathbf{R}^{n}$ is measurable with respect to $\mathcal{B}\left(\mathbf{R}^{n}\right) / \mathcal{B}\left(\mathbf{R}^{n}\right)$, where $\mathcal{P}$ denotes the $\sigma$-field of $\mathcal{F}_{t}$-progressively measurable subsets of $\Omega \times[0, T]$. We assume moreover that $f(\cdot, 0,0) \in L_{\mathbb{F}}^{2}\left(\Omega ; L^{1}\left(0, T ; \mathbf{R}^{n}\right)\right), f(\cdot, \cdot, \cdot)$ is uniformly Lipschitz with respect to its second and third arguments, and $g(\cdot)$ is uniformly Lipschitz continuous.

Definition 3.1. We call that $(y(\cdot), Y(\cdot)) \in L_{\mathbb{F}}^{2}\left(\Omega ; D\left([0, T] ; \mathbf{R}^{n}\right)\right) \times L_{\mathbb{F}}^{2}\left(\Omega \times(0, T) ; \mathbf{R}^{n}\right)$ is a transposition solution to the following BSDE

$$
\left\{\begin{array}{l}
\mathrm{d} y(t)=f(t, y(t), Y(t)) \mathrm{d} t+g(Y(t)) \mathrm{d} W(t), \quad t \in[0, T] \\
y(T)=\xi
\end{array}\right.
$$

if for any $t \in(0, T), \xi \in L_{\mathcal{F}_{T}}^{2}\left(\Omega ; \mathbf{R}^{n}\right)$ and $(u(\cdot), v(\cdot), \eta) \in\left(L_{\mathbb{F}}^{2}\left(\Omega \times(t, T) ; \mathbf{R}^{n}\right)\right)^{2} \times L_{\mathcal{F}_{t}}^{2}\left(\Omega ; \mathbf{R}^{n}\right)$, it holds that

$$
\begin{aligned}
\mathbb{E}\langle x(T), y(T)\rangle & -\mathbb{E}\langle\eta, y(t)\rangle=\mathbb{E} \int_{t}^{T}\langle x(\tau), f(\tau, y(\tau), Y(\tau))\rangle \mathrm{d} \tau \\
& +\mathbb{E} \int_{t}^{T}\langle u(\tau), y(\tau)\rangle \mathrm{d} \tau+\mathbb{E} \int_{t}^{T}\langle v(\tau), g(Y(\tau))\rangle \mathrm{d} \tau,
\end{aligned}
$$

where $x(\cdot)$ is the strong solution to the following forward stochastic differential equation:

$$
\left\{\begin{aligned}
\mathrm{d} x(\tau) & =u(\tau) \mathrm{d} \tau+v(\tau) \mathrm{d} W(\tau), \quad \tau \in[t, T], \\
x(t) & =\eta
\end{aligned}\right.
$$


It is worth mentioning that the equation (3.2) admits a unique transposition solution under $g(Y(t))=Y(t)$ (cf. [9], Thm. 4.1).

The following definition of exact controllability is quoted from [14], Definition 4.2.

Definition 3.2. The system (3.1) is said to be exactly controllable in the transposition sense, if for any $y_{0} \in \mathbf{R}^{n}$ and $\xi \in L_{\mathcal{F}_{T}}^{2}\left(\Omega, \mathbf{R}^{n}\right)$, one can find a $u_{2}(\cdot) \in L_{\mathbb{F}}^{2}\left((0, T) \times \Omega ; \mathbf{R}^{l}\right)$ such that the following BSDE

$$
\left\{\begin{array}{l}
\mathrm{d} y(t)=\left(A y(t)+A_{1} u_{1}(t)+B u_{2}(t)\right) \mathrm{d} t+u_{1}(t) \mathrm{d} W(t), \quad t \in(0, T], \\
y(T)=\xi
\end{array}\right.
$$

admits a transposition solution $\left(y(\cdot), u_{1}(\cdot)\right)$ satisfying $y(0)=y_{0}$.

Let $r>0$ and $y_{d} \in L_{\mathcal{F}_{T}}^{2}\left(\Omega ; \mathbf{R}^{n}\right)$. We consider a norm optimal control problem as follows:

$$
\text { (NP) } \quad \inf _{\left(u_{1}, u_{2}\right) \in \mathcal{U}}\left\|\left(u_{1}, u_{2}\right)\right\|_{\mathcal{H}},
$$

where the admissible control set $\mathcal{U}$ is defined by

$$
\mathcal{U} \equiv\left\{\left(u_{1}, u_{2}\right) \in \mathcal{H} ;\left\|y\left(T ; u_{1}, u_{2}\right)-y_{d}\right\|_{L_{\mathcal{F}_{T}}^{2}\left(\Omega ; \mathbf{R}^{n}\right)} \leq r\right\} .
$$

Note that $\mathcal{U}$ is not empty if the system $(3.1)$ is exactly controllable. We call $\left(\tilde{u}_{1}, \tilde{u}_{2}\right) \in \mathcal{U}$ a norm optimal control pair to the problem (NP) if

$$
\left\|\left(\tilde{u}_{1}, \tilde{u}_{2}\right)\right\|_{\mathcal{H}}=\inf _{\left(u_{1}, u_{2}\right) \in \mathcal{U}}\left\|\left(u_{1}, u_{2}\right)\right\|_{\mathcal{H}} .
$$

To present the main result of this section, we first define a functional $J: L_{\mathcal{F}_{T}}^{2}\left(\Omega ; \mathbf{R}^{n}\right) \rightarrow \mathbf{R}$ by setting

$$
J\left(\varphi_{T}\right)=\frac{1}{2} \mathbb{E} \int_{0}^{T}\left(\left\|B^{*} \varphi(t)\right\|^{2}+\left\|A_{1}^{*} \varphi(t)-\psi(t)\right\|^{2}\right) \mathrm{d} t+r\left\|\varphi_{T}\right\|_{L_{\mathcal{F}_{T}}^{2}\left(\Omega ; \mathbf{R}^{n}\right)}+\mathbb{E}\left\langle\varphi(0), y_{0}\right\rangle-\mathbb{E}\left\langle\varphi_{T}, y_{d}\right\rangle,
$$

where $(\varphi(\cdot), \psi(\cdot))$ solves the following BSDE (in the transposition sense):

$$
\left\{\begin{aligned}
-\mathrm{d} \varphi(t) & =A^{*} \varphi(t) \mathrm{d} t+\psi(t) \mathrm{d} W(t), \quad t \in[0, T] \\
\varphi(T) & =\varphi_{T}
\end{aligned}\right.
$$

with $\varphi_{T} \in L_{\mathcal{F}_{T}}^{2}\left(\Omega ; \mathbf{R}^{n}\right)$. Our main result in this section is stated as follows.

Theorem 3.3. Suppose that (3.1) is exactly controllable in the transposition sense. Let $\hat{\varphi}_{T}$ be the minimizer of the functional $J(\cdot)$ in $L_{\mathcal{F}_{T}}^{2}\left(\Omega ; \mathbf{R}^{n}\right)$. Then $\left(\hat{u}_{1}, \hat{u}_{2}\right)$ defined by

$$
\hat{u}_{1}(t)=A_{1}^{*} \hat{\varphi}(t)-\hat{\psi}(t), \hat{u}_{2}(t)=B^{*} \hat{\varphi}(t), t \in(0, T),
$$

is the unique norm optimal control pair to the problem $(\mathbf{N P})$. Here $(\hat{\varphi}(\cdot), \hat{\psi}(\cdot))$ is the transposition solution to the equation (3.5) with terminal condition $\hat{\varphi}(T)=\hat{\varphi}_{T}$.

Remark 3.4. For the following general control system

$$
\left\{\begin{aligned}
\mathrm{d} y(t) & =(F y(t)+G u(t)) \mathrm{d} t+G_{1} u(t) \mathrm{d} W(t), \quad t \geq 0, \\
y(0) & =y_{0},
\end{aligned}\right.
$$

similar to Definition 3.2, one can define its exact controllability in the transposition sense. By ([14], Rem. 4.4) a necessary condition for (3.6) to be exactly controllable is $R a n k G_{1}=n$. In this case, we can also define the 
norm optimal control problem, and by the same method used in Remark 2.4 the general system (3.6) can be reduced to a standard form like (3.1), i.e.,

$$
\left\{\begin{aligned}
\mathrm{d} y(t) & =\left(F y(t)+A_{1} u_{1}(t)+B u_{2}(t)\right) \mathrm{d} t+u_{1}(t) \mathrm{d} W(t), \quad t \in[0, T], \\
y(0) & =y_{0} .
\end{aligned}\right.
$$

Suppose that $\left(\hat{u}_{1}(\cdot), \hat{u}_{2}(\cdot)\right) \in \mathcal{H}$ and $\hat{y}(\cdot)$ are respectively the optimal control pair and the optimal state of the system $(3.7)$. Then $\hat{u}(\cdot)=M\left(\begin{array}{l}\hat{u}_{1}(\cdot) \\ \hat{u}_{2}(\cdot)\end{array}\right)$ and $\hat{y}(\cdot)$ are respectively the optimal control and state of the system (3.6), where $A_{1}, B, M$ satisfying $G_{1} M=\left(I_{n}, 0\right), G M=\left(A_{1}, B\right)$.

If we verify the existence of minimizers of the functional $J(\cdot)$ defined by (3.4) (see Lem. 3.9 below), then we can prove Theorem 3.3 by the same approach as that in the proof of Theorem 2.3, hence the details are omitted here. To show the existence and uniqueness of minimizers of $J(\cdot)$, we need several lemmas to be given below.

The following lemma, quoted from ([14], Rem. 4.4) provides a Kalman-type rank condition for the system (3.1) to be exactly controllable. It will play a crucial role in proving not only the unique continuation property of solutions to BSDE (3.5) (cf. Lem. 3.8 below), but also the strict convexity of $J(\cdot)$ ( $c f$. Lem. 3.9).

Lemma 3.5 ([14]). The system (3.1) is exactly controllable (in the transposition sense) if and only if

$$
\operatorname{Rank}\left\{B, A B, A_{1} B, A^{2} B, A A_{1} B, A_{1} A B, A_{1}^{2} B, \ldots\right\}=n .
$$

Lemma 3.6. Suppose that the sequence $\left\{\varphi_{T}^{m}\right\}_{m \geq 1} \subset L_{\mathcal{F}_{T}}^{2}\left(\Omega ; \mathbf{R}^{n}\right)$ satisfies

$$
\varphi_{T}^{m} \rightarrow \varphi_{T}^{0} \text { weakly in } L_{\mathcal{F}_{T}}^{2}\left(\Omega ; \mathbf{R}^{n}\right) .
$$

Then

$$
\left(\varphi^{m}(\cdot), \psi^{m}(\cdot)\right) \rightarrow\left(\varphi^{0}(\cdot), \psi^{0}(\cdot)\right) \text { weakly in }\left(L_{\mathbb{F}}^{2}\left((0, T) \times \Omega ; \mathbf{R}^{n}\right)\right)^{2},
$$

where $\left(\varphi^{m}(\cdot), \psi^{m}(\cdot)\right)$ is the transposition solution to the equation $(3.5)$ with $\varphi(T)=\varphi_{T}^{m}$, for each $m \geq 0$.

Proof. For any $(\xi(\cdot), \eta(\cdot)) \in\left(L_{\mathbb{F}}^{2}\left((0, T) \times \Omega ; \mathbf{R}^{n}\right)\right)^{2}$, denote by $x(\cdot)$ the strong solution to the following equation:

$$
\left\{\begin{aligned}
\mathrm{d} x(t) & =(A x(t)+\xi(t)) \mathrm{d} t+\eta(t) \mathrm{d} W(t), \quad t \in[0, T], \\
x(0) & =0 .
\end{aligned}\right.
$$

From the definition of transposition solution (taking the testing equation (3.3) to be (3.10)), we obtain that, for any $m \geq 0$,

$$
\mathbb{E}\left\langle x(T), \varphi_{T}^{m}\right\rangle=\mathbb{E} \int_{0}^{T}\left\langle\xi(t), \varphi^{m}(t)\right\rangle \mathrm{d} t-\mathbb{E} \int_{0}^{T}\left\langle\eta(t), \psi^{m}(t)\right\rangle \mathrm{d} t .
$$

In view of $(3.9),(3.11)$ and the arbitrariness of $(\xi(\cdot), \eta(\cdot))$, as $m$ tends to $+\infty$, we have

$$
\mathbb{E} \int_{0}^{T}\left\langle\xi(t), \varphi^{m}(t)\right\rangle \mathrm{d} t \rightarrow \mathbb{E} \int_{0}^{T}\left\langle\xi(t), \varphi^{0}(t)\right\rangle \mathrm{d} t,
$$

and

$$
\mathbb{E} \int_{0}^{T}\left\langle\eta(t), \psi^{m}(t)\right\rangle \mathrm{d} t \rightarrow \mathbb{E} \int_{0}^{T}\left\langle\eta(t), \psi^{0}(t)\right\rangle \mathrm{d} t
$$

This completes the proof.

Remark 3.7. The previous result is similar to the conclusion (2.11) in Lemma 2.5. But we use a different method (based on the definition of transposition solution) to prove it. The main reason is that Itô's formula cannot be used directly in the present setting. 
Lemma 3.8. Suppose that the system (3.1) is exactly controllable in the transposition sense. Assume that

$$
\mathbb{E} \int_{0}^{T}\left(\left\|B^{*} \varphi(t)\right\|^{2}+\left\|A_{1}^{*} \varphi(t)-\psi(t)\right\|^{2}\right) \mathrm{d} t=0 .
$$

Then

$$
\begin{aligned}
& \varphi(t)=0, \quad \forall t \in[0, T], \\
& \psi(t)=0, \quad \text { a.e. } t \in[0, T],
\end{aligned}
$$

where $(\varphi(\cdot), \psi(\cdot))$ is the transposition solution to the equation (3.5).

Proof. We borrow some idea from [11]. By virtue of Theorem 5.1 from [3], it is clear that the equation (3.5) admits a unique solution $(\varphi(\cdot), \psi(\cdot), M(\cdot)) \in L_{\mathbb{F}}^{2}\left(\Omega ; D\left([0, T] ; \mathbf{R}^{n}\right)\right) \times L_{\mathbb{F}}^{2}\left((0, T) \times \Omega ; \mathbf{R}^{n}\right) \times \mathcal{M}^{2}(0, T)$ satisfying

$$
\varphi(t)-\varphi_{T}=\int_{t}^{T} A^{*} \varphi(s) \mathrm{d} s+\int_{0}^{T} \psi(s) \mathrm{d} W(s)+\int_{0}^{T} d M(s), \forall t \in[0, T],
$$

where $\mathcal{M}^{2}(0, T)$ is the space of square integrable martingales. From (3.12) and the càdlàg property of $\varphi(\cdot)$, it follows that

$$
\begin{aligned}
B^{*} \varphi(t) & =0, \forall t \in[0, T], \\
A_{1}^{*} \varphi(t)-\psi(t) & =0, \text { a.e. } t \in[0, T] .
\end{aligned}
$$

Since $(\varphi(\cdot), \psi(\cdot), M(\cdot))$ is the solution to the equation (3.5), and noting (3.13), we have that for each $t \in[0, T]$,

$$
0=B^{*} \varphi(0)-B^{*} \varphi(t)=\int_{0}^{t} B^{*} A^{*} \varphi(s) \mathrm{d} s+\int_{0}^{t} B^{*} \psi(s) \mathrm{d} W(s)+\int_{0}^{t} B^{*} d M(s) .
$$

From the uniqueness for the decomposition of a semimartingale and the strong orthogonality of the martingales $M(\cdot)$ and $\int_{0}^{\cdot} \psi(s) \mathrm{d} W(s)$, we conclude that

$$
\begin{aligned}
B^{*} A^{*} \varphi(t) & =0, \forall t \in[0, T], \\
B^{*} \psi(t) & =0, \text { a.e. } t \in[0, T] .
\end{aligned}
$$

From (3.13) and (3.14), using the same method we have that

$$
B^{*} A_{1}^{*} \varphi(t)=0, \text { a.e. } t \in[0, T]
$$

Similarly,

$$
\begin{array}{ll}
B^{*} A^{*} A^{*} \varphi(t)=0, & B^{*} A^{*} A_{1}^{*} \varphi(t)=0, \forall t \in[0, T], \\
B^{*} A_{1}^{*} A^{*} \varphi(t)=0, & B^{*} A_{1}^{*} A_{1}^{*} \varphi(t)=0, \forall t \in[0, T],
\end{array}
$$

From (3.13)-(3.15), we obtain that

$$
\left\{B^{*}, B^{*} A^{*}, B^{*} A_{1}^{*}, B^{*} A^{*} A^{*}, B^{*} A_{1}^{*} A_{1}^{*}, B^{*} A^{*} A_{1}^{*}, B^{*} A_{1}^{*} A^{*}, \ldots\right\} \varphi(t)=0, \quad \forall t \in(0, T) .
$$

Since the system (3.1) is exactly controllable in the transposition sense, it follows from Lemma 3.5 that (3.8) holds. Then, $\varphi(t)=0, \forall t \in[0, T]$; hence $\psi(t)=0, M(t)=0$, a.e. $t \in[0, T]$ for the uniqueness of the solution to (3.5). By Remark 4.3 from $[9],(\varphi(\cdot), \psi(\cdot))$, the first two components of the solution to the equation $(3.5)$, coincides with the transposition solution. This completes the proof.

Lemma 3.9. Suppose that the system (3.1) is exactly controllable in the transposition sense. Then the functional $J(\cdot)$ defined by (3.4) admits a unique minimizer $\hat{\varphi}_{T}$ in $L_{\mathcal{F}_{T}}^{2}\left(\Omega ; \mathbf{R}^{n}\right)$. 
Since the proofs are similar to those of Lemmas 2.5 and 2.6 , we only give a sketch.

Sketched Proof of Lemma 3.9. We proceed in the following three steps:

Step 1. The functional $J(\cdot)$ is coercive in $L_{\mathcal{F}_{T}}^{2}\left(\Omega ; \mathbf{R}^{n}\right)$. This statement can be verified by using arguments analogous to the ones described in the proof of Lemma 2.5 with the claim (2.11) replaced by Lemma 3.6.

Step 2. $J(\cdot)$ is strictly convex. Its proof is analogous to the first part of the proof of Lemma 2.6 with the use of Proposition 2.2 replaced by that of Lemma 3.8.

Step 3. $J(\cdot)$ admits a unique minimizer $\hat{\varphi}_{T}$ in $L_{\mathcal{F}_{T}}^{2}\left(\Omega ; \mathbf{R}^{n}\right)$. The proof of this assertion is similar to the second part of the proof of Lemma 2.6.

Acknowledgements. The authors gratefully acknowledge Professor Xu Zhang for his valuable suggestions during this work; they also thank the anonymous referees for pointing out references $[7,8]$ and their constructive comments for improving the quality of this paper.

\section{REFERENCES}

[1] R. Buckdahn, M. Quincampoix and G. Tessitore, A characterization of approximately controllable linear stochastic differential equations, in Stoch. Partial Differ. Equ. Appl., edited by G. Da Prato and L. Tubaro. Chapman \& Hall, Boca Raton (2006) $253-260$.

[2] M. Ehrhardt and W. Kliemann, Controllability of linear stochastic systems. Syst. Control Lett. 2 (1982) $145-153$.

[3] N. El Karoui, S. Peng and M.C. Quenez, Backward stochastic differential equations in finance. Math. Finance 7 (1997) 1-71.

[4] H.O. Fattorini, Infinite Dimensional Linear Control Systems, The Time Optimal and Norm Optimal Problems. Elsevier, Amsterdam (2005).

[5] D. Goreac, A Kalman-type condition for stochastic approximate controllability. C.R. Math. Acad. Sci. Paris 346 (2008), $183-188$.

[6] D. Goreac, A note on the controllability of jump diffusions with linear coefficients. IMA J. Math. Control Inform. 29 (2012) 427-435.

[7] S. Ji and X.Y. Zhou, A maximum principle for stochastic optimal control with terminal state constraints, and its applications. Commun. Inform. Syst. 6 (2006) 321-337.

[8] A.E.B. Lim and X.Y. Zhou, Stochastic optimal LQR control with integral quadratic constraints and indefinite control weights. IEEE Trans. Automat. Control. 44 (1999) 1359-1369.

[9] Q. Lü and X. Zhang, Well-posedness of backward stochastic differential equations with general filtration. J. Differ. Equ. 254 (2013) 3200-3227.

[10] E. Pardoux and S. Peng, Adapted solution of a backward stochastic differential equation. Systems Control Lett. 14 (1990) 55-61.

[11] S. Peng, Backward stochastic differential equation and exact controllability of stochastic control systems. Prog. Nat. Sci. 4 (1994) 274-284.

[12] S. Tang and X. Li, Necessary conditions for optimal control of stochastic systems with random jumps. SIAM J. Control Optim. 32 (1994) 1447-1475.

[13] G. Wang and E. Zuazua, On the equivalence of minimal time and minimal norm controls for heat equations. SIAM J. Control Optim. 50 (2012), 2938-2958.

[14] Y. Wang, BSDEs with general filtration driven by Lévy processes, and an application in stochastic controllability. Syst. Control Lett. 62 (2013) 242-247.

[15] J. Yong and X. Y. Zhou, Stochastic Controls: Hamiltonian Systems and HJB Equations. Springer, New York (1999).

[16] J. Zabczyk, Controllability of stochastic linear systems. Syst. Control Lett. 1 (1981) 25-31.

[17] E. Zuazua, Controllability and observability of partial differential equations: some results and open problems, in vol. 3, Handb. Differ. Equ.: Evol. Differ. Equ. Elsevier Science, New York (2006) 527-621. 